\title{
New results on the existence of solutions of boundary value problems for singular fractional differential systems with impulse effects
}

\author{
Yuji Liu \\ Department of Mathematics, Guangdong University of Finance and Economics, Guangzhou 510320, P.R.China \\ E-mail: liuyuji888@sohu.com
}

\begin{abstract}
Results on the existence of solutions to a new class of impulsive singular fractional differential systems with multiple base points are established. The assumptions imposed on the nonlinearities, see ((C) and (D) in Theorem 3.1), are weaker than known ones, (i.e., (A) in Introduction section). The analysis relies on the well known fixed point theorems. An example is given to illustrate the efficiency of the main theorems. The investigation shows that these results and methods are helpful for study in the nonlinear area and the numerical simulation, especially for study in the the numerical solution of a fractional differential equation with multiple base points with or without impulse effects. A section "Conclusions" is given with future work research directions.
\end{abstract}

2010 Mathematics Subject Classification. 92D25. 34A37, 34K15

Keywords. Singular fractional differential system, impulsive boundary value problem, Riemann-Liouville fractional differential equation with multiple base points, fixed point theorem.

\section{Introduction}

Fractional differential equations is a generalization of ordinary differential equations to arbitrary non integer orders. The origin of fractional calculus goes back to Newton and Leibniz in the seventieth century. Recent investigations have shown that many physical systems can be represented more accurately through fractional derivative formulation.

The theory of impulsive differential equations describes processes which experience a sudden change of their state at certain moments. Processes with such a character arise naturally and often, for example, phenomena studied in physics, chemical technology, population dynamics, biotechnology and economics. For an introduction of the basic theory of impulsive differential equation.

In recent papers [1-17], the authors studied the existence or uniqueness of positive solutions or solutions of boundary value problems for the impulsive fractional differential equations. While the existence of solutions of impulsive boundary value problems for Riemann-Liouville fractional differential equations has not been given up to now, the research proceeds slowly and appears some new difficulties.

In [1], the following boundary value problem for fractional differential equation with the base point $t=0$ was discussed

$$
\left\{\begin{array}{l}
D_{0^{+}}^{\alpha} u(t)-\lambda u(t)=f(t, u(t)), \quad t \in(0,1], 0<\alpha \leq 1, \\
\lim _{t \rightarrow 0} t^{1-\alpha} u(t)=u(1),
\end{array}\right.
$$

Tbilisi Mathematical Journal 8(2) (2015), pp. 1-22

Tbilisi Centre for Mathematical Sciences.

Received by the editors: 14 August 2014

Accepted for publication: 20 September 2014 
where $D_{0^{+}}^{\alpha}$ is the Riemann-Liouville fractional derivative of order $\alpha, f$ is continuous and $\lambda \in R$. The existence and uniqueness of solutions of $\operatorname{BVP}(2)$ are established under some assumptions by using Banach contraction principle. One of the main assumptions in [1] is as follows (see Theorem 4.3 and Remark 4.4 in [1]):

(B). there exist positive numbers $M$ and $k>0$ such that $|f(t, x)| \leq M$ and $|f(t, u)-f(t, v)| \leq$ $k|u-v|$ hold for all $t \in[0,1]$ and $u, v \in R$ or

(C). for each $u_{0} \in C_{1-\alpha}[0,1]$ fixed, there exists $k_{u_{0}}>0$ such that

$$
\left|f(t, u)-f\left(t, u_{0}(t)\right)\right| \leq k_{u_{0}}\left|u-u_{0}(t)\right|, t \in[0,1], u \in R .
$$

In [18], the authors studied the existence and uniqueness of solutions of the following periodic boundary value problem of the impulsive fractional differential equation with a single base point $t=0$

$$
\left\{\begin{array}{l}
D_{0^{+}}^{\alpha} u(t)-\lambda u(t)=f(t, u(t)), \quad t \in(0,1], t \neq t_{1} \in(0,1), 0<\alpha \leq 1, \\
\lim _{t \rightarrow 0} t^{1-\alpha} u(t)=u(1), \\
\lim _{t \rightarrow t_{1}^{+}}\left[t-t_{1}\right]^{1-\alpha}\left[u(t)-u\left(t_{1}\right)\right]=I\left(u\left(t_{1}\right)\right),
\end{array}\right.
$$

where $D_{0^{+}}^{\alpha}$ is the Riemann-Liouville fractional derivative of order $\alpha, I: R \rightarrow R$ is continuous, $f:[0,1] \times R \rightarrow R$ is continuous, $\lambda \in R$ is a constant. The existence and uniqueness of solutions of BVP(1) are established under some assumptions by using Banach contraction principle. One of the main assumptions in [18] is as follows:

(A). there exist positive numbers $M$ and $m$ such that

$$
|f(t, x)| \leq M,|I(x)| \leq m, t \in[0,1], x \in R .
$$

In recent paper [22], the following periodic boundary value problem of impulsive fractional differential equation with multiple base points

$$
\left\{\begin{array}{l}
D_{t_{i}^{+}}^{\alpha} u(t)-\lambda u(t)=f(t, u(t)), \quad t \in\left(t_{i}, t_{i+1}\right], i=0,1, \\
\lim _{t \rightarrow 0} t^{1-\alpha} u(t)=u(1), \\
\lim _{t \rightarrow t_{1}^{+}}\left[t-t_{1}\right]^{1-\alpha}\left[u(t)-u\left(t_{1}\right)\right]=I_{1}\left(u\left(t_{1}\right)\right),
\end{array}\right.
$$

was discussed, where where $\alpha \in(0,1) 0=t_{0}<t_{1}<t_{2}=1, D_{0^{+}}^{\alpha}$ is the Riemann-Liouville fractional derivative of order $\alpha, I_{1} \in C(R, R), f$ is s continuous at every point $(t, u) \in\left(t_{i}, t_{i+1}\right] \times R$. The existence and uniqueness of solutions of (3) were proved under the assumption (B).

In this paper, we discuss the boundary value problem of the impulsive singular fractional differential system with multiple base points

$$
\left\{\begin{array}{l}
D_{*}^{\alpha} x(t)-\lambda x(t)=p(t) f(t, x(t), y(t)), \quad t \in(0,1), t \neq t_{1} \\
D_{*}^{\beta} y(t)-\mu y(t)=q(t) g(t, x(t), y(t)), \quad t \in(0,1), t \neq t_{1}, \\
x(1)-\lim _{t \rightarrow 0} t^{1-\alpha} x(t)=\int_{0}^{1} \varphi(s) G(s, x(s), y(s)) d s, \\
y(1)-\lim _{t \rightarrow 0} t^{1-\beta} y(t)=\int_{0}^{1} \psi(s) H(s, x(s), y(s)) d s, \\
\lim _{t \rightarrow t_{1}^{+}}\left[t-t_{1}\right]^{1-\alpha} x(t)=I\left(t_{1}, x\left(t_{1}\right), y\left(t_{1}\right)\right), \\
\lim _{t \rightarrow t_{1}^{+}}\left[t-t_{1}\right]^{1-\beta} y(t)=J\left(t_{1}, x\left(t_{1}\right), y\left(t_{1}\right)\right) .
\end{array}\right.
$$


where

$(\bullet) 0<\alpha, \beta<1, \lambda, \mu \in R, D_{*}^{\alpha}$ ( or $D_{*}^{\beta}$ ) is the Riemann-Liouville fractional derivative of order $\alpha$ ( or $\beta$ ) with the base point $*$,

$(\bullet) 0=t_{0}<t_{1}<t_{2}=1, I, J:(0,1) \times R^{2} \rightarrow R$ are continuous functions,

$(\bullet) \varphi, \psi:(0,1) \rightarrow R$ satisfy that

$$
\left.\varphi\right|_{\left(0, t_{1}\right)},\left.\psi\right|_{\left(0, t_{1}\right)} \in L^{1}\left(0, t_{1}\right),\left.\varphi\right|_{\left(t_{1}, 1\right)},\left.\psi\right|_{\left(t_{1}, 1\right)} \in L^{1}\left(t_{1}, 1\right),
$$

$(\bullet) p, q:\left(t_{0}, t_{1}\right) \bigcup\left(t_{1}, t_{2}\right) \rightarrow R$ satisfy that there exist constants $k_{1}>-1,-\alpha<l_{1} \leq 0$ with $\alpha+k_{1}+l_{1} \geq 0, k_{2}>-1,-\beta<l_{2} \leq 0$ with $\beta+k_{2}+l_{2} \geq 0$ such that

$$
|p(t)| \leq\left(t-t_{i}\right)^{k_{1}}\left(t_{i+1}-t\right)^{l_{1}},|q(t)| \leq\left(t-t_{i}\right)^{k_{2}}\left(t_{i+1}-t\right)^{l_{2}} \text { for } t \in\left(t_{i}, t_{i+1}\right), i=0,1,
$$

$(\bullet) f, g, G, H, I, J$ defined on $(0,1] \times R \times R$ are Caratheodory functions (see Definition 2.5), all of which may be singular at the points $t=0, t_{1}, 1$.

A pair of functions $(x, y)$ defined on $(0,1)$ is called a solution of $\operatorname{BVP}(4)$, if

$$
\begin{aligned}
& \left.x\right|_{\left(t_{i}, t_{i+1}\right]} \in C^{0}\left(0, t_{1}\right],\left.\quad y\right|_{\left(t_{i}, t_{i+1}\right]} \in C^{0}\left(0, t_{1}\right], i=0,1, \\
& \left.D_{t_{i}^{+}}^{\alpha} x\right|_{\left(t_{i}, t_{i+1}\right]} \in L^{1}\left(t_{i}, t_{i+1}\right],\left.\quad D_{t_{i}^{+}}^{\beta} y\right|_{\left(t_{i}, t_{i+1}\right]} \in L^{1}\left(t_{i}, t_{i+1}\right], i=0,1,
\end{aligned}
$$

there exist the $\operatorname{limits}_{\lim _{t \rightarrow t_{i}^{+}}}\left(t-t_{i}\right)^{1-\alpha} x(t), \lim _{t \rightarrow t_{i}^{+}}\left(t-t_{i}\right)^{1-\beta} y(t), \quad i=0,1$,

and $x, y$ satisfy all equations in (4).

We obtain the results on the existence of at least one solution of $\operatorname{BVP}(4)$. An example is given to illustrate the efficiency of the main theorem. The results in this paper generalize those ones in $[1,18]$, i.e., assumptions (A) or (B) is replaced by the weaker ones (see (C) and (D) in Section 3). Both $p$ and $q$ maybe singular at $t=0, t_{1}, t_{2}$. The impulse functions in this paper are different from those ones in known paper [18,22].

In the left and right fractional derivatives $D_{a^{+}}^{\alpha} x$ and $D_{b^{-}}^{\alpha} x, a$ is called a left base point and $b$ right base point. Both $a$ and $b$ are called base points of fractional derivatives. An FDE containing more than one base point is called a multiple base points FDE. An FDE containing only one base point is called a single base point FDE. It is easy to see that the fractional differential equations in (1) contains only one base point $t=0$. So the problem we are concerned is a new one. In $[1,18,22]$, the assumptions imposed on the nonlinearities are (A), (B) and (C). But in this paper the assumptions imposed on $f, g$ in (4) involving sup-multiplicative-like functions which do not satisfy (A), (B) and (C) (see Definition 2.6). So our results are new.

The remainder of this paper is as follows: in Section 2, we present preliminary results. In Section 3 , the main theorems and their proof are given. In Section 4, an example is given to illustrate the main results.

\section{Preliminary results}

For the convenience of the readers, we firstly present the necessary definitions from the fractional calculus theory. These definitions and results can be found in the literatures [8]. Let the Gamma function $\mathbf{B}(p, q)$ and the beta functions $\Gamma(\alpha)$ and be defined by

$$
\Gamma(\alpha)=\int_{0}^{+\infty} x^{\alpha-1} e^{-x} d x, \quad \mathbf{B}(p, q)=\int_{0}^{1} x^{p-1}(1-x)^{q-1} d x .
$$


The Mitag-Leffler function (see [8]) is defined by $E_{\alpha, \alpha}(\lambda)=\sum_{k=0}^{\infty} \frac{\lambda^{k}}{\Gamma((k+1) \alpha)}$. We note that $E_{\alpha, \alpha}(x)>0$ for every $x \in R$ and $E_{\alpha, \alpha}(x)$ is strictly increasing in $x[21], \lim _{x \rightarrow 0} E_{\alpha, \alpha}(x)=\frac{1}{\Gamma(\alpha)}$. Then for $x>0$ we have that $E_{\alpha, \alpha}(-x)<E_{\alpha, \alpha}(0)=\frac{1}{\Gamma(\alpha)}<E_{\alpha, \alpha}(x)$.

Definition 2.1[8]. Let $a \geq 0$. The Riemann-Liouville fractional integral of order $\alpha>0$ of a function $g:(a, \infty) \rightarrow R$ is given by

$$
I_{a^{+}}^{\alpha} g(t)=\frac{1}{\Gamma(\alpha)} \int_{a}^{t}(t-s)^{\alpha-1} g(s) d s,
$$

provided that the right-hand side exists, $a$ is called a base point.

Definition 2.2[8]. Let $a \geq 0$. The Riemann-Liouville fractional derivative of order $\alpha>0$ of a function $g:(a, \infty) \rightarrow R$ is given by

$$
D_{a^{+}}^{\alpha} g(t)=\frac{1}{\Gamma(n-\alpha)} \frac{d^{n}}{d t^{n}} \int_{a}^{t} \frac{g(s)}{(t-s)^{\alpha-n+1}} d s,
$$

where $n-1 \leq \alpha<n$, provided that the right-hand side exists, $a$ is called a base point.

Definition 2.3. Let $E$ and $Z$ be Banach spaces. $L: D(L) \subset E \rightarrow Z$ is called a Fredholm operator of index zero if $\operatorname{Im} L$ is closed in $E$ and $\operatorname{dim} \operatorname{Ker} L=\operatorname{co} \operatorname{dim} \operatorname{Im} L<+\infty$.

It is easy to see that if $L$ is a Fredholm operator of index zero, then there exist the projectors $P: E \rightarrow E$, and $Q: Z \rightarrow Z$ such that

$$
\operatorname{Im} P=\operatorname{Ker} L, \operatorname{Ker} Q=\operatorname{Im} L, \quad E=\operatorname{Ker} L \oplus \operatorname{Ker} P, Z=\operatorname{Im} L \oplus \operatorname{Im} Q .
$$

If $L: D(L) \subset E \rightarrow Z$ is called a Fredholm operator of index zero, the inverse of

$$
\left.L\right|_{D(L) \cap \operatorname{Ker} P}: D(L) \cap \operatorname{Ker} P \rightarrow \operatorname{Im} L
$$

is denoted by $K_{p}$.

Definition 2.4. Suppose that $L: D(L) \subset E \rightarrow Z$ is called a Fredholm operator of index zero. The continuous map $N: E \rightarrow Z$ is called $L$-compact if $Q N(\bar{\Omega})$ is bounded and $K_{p}(I-Q) N(\bar{\Omega})$ is compact for each nonempty open subset $\Omega$ of $X$ satisfying $D(L) \cap \bar{\Omega} \neq \varnothing$.

To obtain the main results, we need the abstract existence theorem.

Lemma 2.1 [19] Leray-Schauder Nonlinear Alternative. Let $E, Z$ be Banach spaces and $L: D(L) \bigcap E \rightarrow Z$ a Fredholm operator of index zero with $\operatorname{Ker} L=\{0 \in E\}, N: E \rightarrow Z$ $L$-compact. Suppose $\Omega$ is a nonempty open subset of $X$ satisfying $D(L) \cap \bar{\Omega} \neq \varnothing$. Then either there exists $x \in \partial \Omega$ and $\theta \in(0,1)$ such that $L x=\theta N x$ or there exists $x \in \bar{\Omega}$ such that $L x=N x$.

Definition 2.5. We call $F:(0,1) \times R^{2} \rightarrow R$ an Caratheodory function if it satisfies the followings:

(i) $t \rightarrow F\left(t,\left(t-t_{i}\right)^{\alpha-1} u,\left(t-t_{i}\right)^{\beta-1} v\right)(i=0,1)$ are measurable on $\left(t_{i}, t_{i+1}\right](i=0,1)$ and there exist the $\operatorname{limits}_{t \rightarrow t_{i}^{+}} F\left(t,\left(t-t_{i}\right)^{\alpha-1} u,\left(t-t_{i}\right)^{\beta-1} v\right), i=0,1$ 
(ii) $(x, y) \rightarrow F\left(t,\left(t-t_{i}\right)^{\alpha-1} x,\left(t-t_{i}\right)^{\beta-1} y\right)(i=0,1)$ are continuous on $R^{2}$ for all $t \in(0,1]$,

(iii) for each $r>0$ there exists $M_{r}>0$ such that $\left|F\left(t,\left(t-t_{i}\right)^{\alpha-1} x,\left(t-t_{i}\right)^{\beta-1} y\right)\right| \leq M_{r}$ for all $t \in\left(t_{i}, t_{i+1}\right]$ and $|x|,|y| \leq r(\mathrm{i}=0,1)$.

Definition 2.6[20]. An odd homeomorphism $\Phi$ of the real line $R$ onto itself is called a supmultiplicative-like function if there exists a homeomorphism $\omega$ of $[0,+\infty)$ onto itself which supports $\Phi$ in the sense that for all $v_{1}, v_{2} \geq 0$ it holds

$$
\Phi\left(v_{1} v_{2}\right) \geq \omega\left(v_{1}\right) \Phi\left(v_{2}\right) .
$$

$\omega$ is called the supporting function of $\Phi$.

Remark 2.1. Note that any sup-multiplicative function is sup-multiplicative-like function. Also any function of the form

$$
\Phi(u):=\sum_{j=0}^{k} c_{j}|u|^{j} u, \quad u \in R
$$

is sup-multiplicative-like, provided that $c_{j} \geq 0$. Here a supporting function is defined by $\omega(u):=$ $\min \left\{u^{k+1}, \quad u\right\}, u \geq 0$.

Remark 2.2. It is clear that a sup-multiplicative-like function $\Phi$ and any corresponding supporting function $\omega$ are increasing functions vanishing at zero and moreover their inverses $\Phi^{-1}$ and $\nu$ respectively are increasing and such that

$$
\Phi^{-1}\left(w_{1} w_{2}\right) \leq \nu\left(w_{1}\right) \Phi^{-1}\left(w_{2}\right)
$$

for all $w_{1}, w_{2} \geq 0$ and $\nu$ is called the supporting function of $\Phi^{-1}$.

To apply Lemma 2.1, we need to define the Banach spaces $E$ and $Z$. We use the Banach spaces

$$
X=\left\{x:(0,1] \rightarrow R: \begin{array}{c}
\left.x\right|_{\left(0, t_{1}\right]} \in C^{0}\left(0, t_{1}\right],\left.x\right|_{\left(t_{1}, 1\right]} \in C^{0}\left(t_{1}, 1\right] \\
\text { there exist the limits } \\
\lim _{t \rightarrow 0^{+}} t^{1-\alpha} x(t), \lim _{t \rightarrow t_{1}^{+}}\left(t-t_{1}\right)^{1-\alpha} x(t)
\end{array}\right\}
$$

with the norm

$$
\begin{aligned}
& \|x\|=\|x\|_{\infty}=\max \left\{\sup _{t \in\left(0, t_{1}\right]} t^{1-\alpha}|x(t)|, \sup _{t \in\left(t_{1}, 1\right]}\left(t-t_{1}\right)^{1-\alpha}|x(t)|\right\} \\
& Y=\left\{\begin{array}{cc} 
& \left.y\right|_{\left(0, t_{1}\right]} \in C^{0}\left(0, t_{1}\right],\left.y\right|_{\left(t_{1}, 1\right]} \in C^{0}\left(t_{1}, 1\right] \\
\text { there exist the limits } & \\
& \lim _{t \rightarrow 0^{+}} t^{1-\beta} y(t), \lim _{t \rightarrow t_{1}^{+}}\left(t-t_{1}\right)^{1-\beta} y(t)
\end{array}\right\}
\end{aligned}
$$

with the norm

$$
\|y\|=\|y\|_{\infty}=\max \left\{\sup _{t \in\left(0, t_{1}\right]} t^{1-\beta}|y(t)|, \sup _{t \in\left(t_{1}, 1\right]}\left(t-t_{1}\right)^{1-\beta}|y(t)|\right\}
$$


$L^{1}[0,1]$ with the norm

$$
\|u\|_{1}=\int_{0}^{1}|u(s)| d s .
$$

Choose $E=X \times Y$ with the norm

$$
\|(x, y)\|=\max \left\{\|x\|_{\infty},\|y\|_{\infty}\right\} .
$$

Choose $Z=L^{1}(0,1) \times L^{1}(0,1) \times R^{4}$ with the norm

$$
\left\|\left(\begin{array}{c}
u \\
v \\
c \\
d \\
c \\
d
\end{array}\right)^{T}\right\|=\|(u, v, a, b, c, d)\|=\max \left\{\|u\|_{1},\|v\|_{1},|a|,|b|,|c|,|d|\right\} .
$$

Define $L$ to be the linear operator from $D(L) \bigcap E$ to $Z$ with

$$
D(L)=\left\{(x, y) \in E: D_{t_{i}^{+}}^{\alpha} x, D_{t_{i}^{+}}^{\beta} y \in L^{1}\left(t_{i}, t_{i+1}\right]\right\}
$$

and

$$
L(x, y)(t)=\left(\begin{array}{c}
D_{t_{i}^{+}}^{\alpha} x(t)-\lambda x(t) \\
D_{t_{i}^{+}}^{\beta} y(t)-\mu y(t) \\
x(1)-\lim _{t \rightarrow 0} t^{1-\alpha} x(t) \\
y(1)-\lim _{t \rightarrow 0} t^{1-\beta} y(t) \\
\lim _{t \rightarrow t_{1}^{+}}\left[t-t_{1}\right]^{1-\alpha} x(t) \\
\lim _{t \rightarrow t_{1}^{+}}\left[t-t_{1}\right]^{1-\beta} y(t)
\end{array}\right)^{T}
$$

for $(x, y) \in E$. Define $N: E \rightarrow Z$ by

$$
N(x, y)(t)=\left(\begin{array}{c}
p(t) f(t, x(t), y(t)) \\
q(t) g(t, x(t), y(t)) \\
\int_{0}^{1} \varphi(s) G(t, x(t), y(t)) d t \\
\int_{0}^{1} \psi(s) H(t, x(t), y(t)) d t \\
I\left(t_{1}, x\left(t_{1}\right), y\left(t_{1}\right)\right) \\
J\left(t_{1}, x\left(t_{1}\right), y\left(t_{1}\right)\right)
\end{array}\right)^{T} \quad \text { for }(x, y) \in E
$$

Then BVP(4) can be written as

$$
L(x, y)=N(x, y), \quad(x, y) \in E \bigcap D(L) .
$$

Lemma 2.2. Suppose that $f, g, G, H, I, J$ are Caratheodory functions. Then $L$ is a Fredholm operator with index zero and $N: X \rightarrow X$ is $L$-compact.

Proof. To prove that $L$ is a is a Fredholm operator with index zero, we should do the following three steps. 
Step (i) Prove that $\operatorname{Ker} L=\{(0,0) \in E\}$.

We know that $(x, y) \in \operatorname{Ker} L$ if and only if

$$
\left\{\begin{array}{l}
D_{t_{i}^{+}}^{\alpha} x(t)-\lambda x(t)=0, \\
D_{t_{i}^{+}}^{\beta} y(t)-\mu y(t)=0, \\
x(1)-\lim _{t \rightarrow 0} t^{1-\alpha} x(t)=0, \\
y(1)-\lim _{t \rightarrow 0} t^{1-\beta} y(t)=0, \\
\lim _{t \rightarrow t_{1}}\left[t-t_{1}\right]^{1-\alpha} x(t)=0, \\
\lim _{t \rightarrow t_{1}^{+}}\left[t-t_{1}\right]^{1-\beta} y(t)=0 .
\end{array}\right.
$$

From Lemma 2.1 in [3], $D_{t_{0}^{+}}^{\alpha} x(t)-\lambda x(t)=0$ implies that there exists $c_{0} \in R$ such that $x(t)=$ $c_{0} \Gamma(\alpha) t^{\alpha-1} E_{\alpha, \alpha}\left(\lambda t^{\alpha}\right)$ on $\left(t_{0}, t_{1}\right]$. Now $D_{t_{1}^{+}}^{\alpha} x(t)-\lambda x(t)=0$ implies that there exists $c_{1} \in R$ such that $x(t)=c_{1} \Gamma(\alpha)\left(t-t_{1}\right)^{\alpha-1} E_{\alpha, \alpha}\left(\lambda\left(t-t_{1}\right)^{\alpha}\right)$ on $\left(t_{1}, t_{2}\right]$. It follows from $x(1)-\lim _{t \rightarrow 0} t^{1-\alpha} x(t)=0$ that $c_{1} \Gamma(\alpha)\left(1-t_{1}\right)^{\alpha-1} E_{\alpha, \alpha}\left(\lambda\left(1-t_{1}\right)^{\alpha}\right)=c_{0} \cdot \lim _{t \rightarrow t_{1}}\left[t-t_{1}\right]^{1-\alpha} x(t)=0$ implies that $c_{1}=0$. Hence we get $c_{0}=0$. Then $x(t)=0$ on $[0,1]$. Similarly we get $y(t)=0$ on $[0,1]$. Thus $\operatorname{Ker} L=\{(0,0) \in E \in R\}$.

Step (ii) Prove that $\operatorname{Im} L=Z$.

For $(u, v, a, b, c, d) \in Z$, we know that $(u, v, a, b, c, d) \in \operatorname{Im} L$ if and only if there exist $(x, y) \in$ $E \bigcap D(L)$ such that

$$
\left\{\begin{array}{l}
D_{t_{i}^{+}}^{\alpha} x(t)-\lambda x(t)=u(t), t \in\left(t_{i}, t_{i+1}\right], i=0,1 \\
D_{t_{i}^{+}}^{\beta} y(t)-\mu y(t)=v(t), t \in\left(t_{i}, t_{i+1}\right], i=0,1 \\
x(1)-\lim _{t \rightarrow 0} t^{1-\alpha} x(t)=a, \quad y(1)-\lim _{t \rightarrow 0} t^{1-\beta} y(t)=b \\
\lim _{t \rightarrow t_{1}}\left[t-t_{1}\right]^{1-\alpha} x(t)=c, \quad \lim _{t \rightarrow t_{1}^{+}}\left[t-t_{1}\right]^{1-\beta} y(t)=d
\end{array}\right.
$$

We write the general solution of $D_{t_{i}^{+}}^{\alpha} x(t)-\lambda x(t)=u(t)$ by

$$
\begin{aligned}
& x(t)=c_{i} \Gamma(\alpha)\left(t-t_{i}\right)^{\alpha-1} E_{\alpha, \alpha}\left(\lambda\left(t-t_{i}\right)^{\alpha}\right) \\
& +\int_{t_{i}}^{t}(t-s)^{\alpha-1} E_{\alpha, \alpha}\left(\lambda(t-s)^{\alpha}\right) u(s) d s, t \in\left(t_{i}, t_{i+1}\right], i=0,1 .
\end{aligned}
$$

where $\lim _{t \rightarrow 0^{+}} t^{1-\alpha} x(t)=c_{0}, \lim _{t \rightarrow t_{1}^{+}}\left(t-t_{1}\right)^{1-\alpha} x(t)=c_{1}$. Use $x(1)-\lim _{t \rightarrow 0} t^{1-\alpha} x(t)=a$, we get

$$
c_{1} \Gamma(\alpha)\left(1-t_{1}\right)^{\alpha-1} E_{\alpha, \alpha}\left(\lambda\left(1-t_{1}\right)^{\alpha}\right)+\int_{t_{1}}^{1}(1-s)^{\alpha-1} E_{\alpha, \alpha}\left(\lambda(1-s)^{\alpha}\right) u(s) d s-c_{0}=a .
$$


Use $\lim _{t \rightarrow t_{1}}\left[t-t_{1}\right]^{1-\alpha} x(t)=c$, we get $c_{1}=c$. Hence

$$
x(t)=\left\{\begin{array}{l}
c \Gamma(\alpha)^{2}\left(1-t_{1}\right)^{\alpha-1} E_{\alpha, \alpha}\left(\lambda\left(1-t_{1}\right)^{\alpha}\right) t^{\alpha-1} E_{\alpha, \alpha}\left(\lambda t^{\alpha}\right)-a \Gamma(\alpha) t^{\alpha-1} E_{\alpha, \alpha}\left(\lambda t^{\alpha}\right) \\
+\Gamma(\alpha) t^{\alpha-1} E_{\alpha, \alpha}\left(\lambda t^{\alpha}\right) \int_{t_{1}}^{1}(1-s)^{\alpha-1} E_{\alpha, \alpha}\left(\lambda(1-s)^{\alpha}\right) u(s) d s \\
+\int_{0}^{t}(t-s)^{\alpha-1} E_{\alpha, \alpha}\left(\lambda(t-s)^{\alpha}\right) u(s) d s, t \in\left(0, t_{1}\right], \\
c \Gamma(\alpha)\left(t-t_{1}\right)^{\alpha-1} E_{\alpha, \alpha}\left(\lambda\left(t-t_{1}\right)^{\alpha}\right) \\
+\int_{t_{1}}^{t}(t-s)^{\alpha-1} E_{\alpha, \alpha}\left(\lambda(t-s)^{\alpha}\right) u(s) d s, t \in\left(t_{1}, 1\right] .
\end{array}\right.
$$

Similarly we can get that

$$
y(t)=\left\{\begin{array}{l}
d \Gamma(\beta)^{2}\left(1-t_{1}\right)^{\beta-1} E_{\beta, \beta}\left(\mu\left(1-t_{1}\right)^{\beta}\right) t^{\beta-1} E_{\beta, \beta}\left(\mu t^{\beta}\right)-b \Gamma(\beta) t^{\beta-1} E_{\beta, \beta}\left(\mu t^{\beta}\right) \\
+\Gamma(\beta) t^{\beta-1} E_{\beta, \beta}\left(\mu t^{\beta}\right) \int_{t_{1}}^{1}(1-s)^{\beta-1} E_{\beta, \beta}\left(\mu(1-s)^{\beta}\right) v(s) d s \\
+\int_{0}^{t}(t-s)^{\beta-1} E_{\beta, \beta}\left(\mu(t-s)^{\beta}\right) v(s) d s, t \in\left(0, t_{1}\right], \\
d \Gamma(\beta)\left(t-t_{1}\right)^{\beta-1} E_{\beta, \beta}\left(\mu\left(t-t_{1}\right)^{\beta}\right) \\
+\int_{t_{1}}^{t}(t-s)^{\beta-1} E_{\beta, \beta}\left(\mu(t-s)^{\beta}\right) v(s) d s, t \in\left(t_{1}, 1\right] .
\end{array}\right.
$$

One can show that if $(x, y)$ satisfies $(9)$ and $(10)$, then $(x, y)$ is a solution of (8). Then (8) has aa unique solution $(x, y)$ defined by (9) and (10). It is easy to show that $(x, y) \in E \bigcap D(L)$. Then $\operatorname{Im} L=Z$.

Step (iii) Prove that $\operatorname{Im} L$ is closed in $X$ and $\operatorname{dim} \operatorname{Ker} L=\operatorname{co} \operatorname{dim} \operatorname{Im} L<+\infty$..

From Step (ii) $\operatorname{Im} L=Z$ is closed in $Z$. It follows from $\operatorname{Ker} L=\{(0,0) \in E\}$ that $\operatorname{dim} \operatorname{Ker} L=0$. Define the projector $P: E \rightarrow E$ by

$$
P(x, y)(t)=(0,0) \text { for }(x, y) \in E .
$$

It is easy to prove that

$$
\operatorname{Im} P=\operatorname{Ker} L, \quad E=\operatorname{Ker} L \oplus \operatorname{Ker} P .
$$

Define the projector $Q: Z \rightarrow Z$ by

$$
Q(u, v, a, b, c, d)(t)=(0,0,0,0,0,0)
$$

for $(u, v, a, b, c, d) \in Z$.

It is easy to show that

$$
\operatorname{Im} L=\operatorname{Ker} Q, \quad Z=\operatorname{Im} Q \oplus \operatorname{Im} L .
$$

From above discussion, we see that $\operatorname{dim} \operatorname{Ker} L=c o \operatorname{dim} \operatorname{Im} L=0<+\infty$. So $L$ is a Fredholm operator of index zero. 
Now, we prove that $N$ is $L$-compact. The proof is standard and is divided into three steps.

Step (i) We prove that $N$ is continuous.

Let $\left(x_{n}, y_{n}\right) \in E$ with $\left(x_{n}, y_{n}\right) \rightarrow\left(x_{0}, y_{0}\right)$ as $n \rightarrow \infty$. We will show that $N\left(x_{n}, y_{n}\right) \rightarrow N\left(x_{0}, y_{0}\right)$ as $n \rightarrow \infty$.

In fact, there exists $r>0$ such that $\left\|\left(x_{n}, y_{n}\right)\right\| \leq r$ for all $n$. By

$$
N\left(x_{n}, y_{n}\right)(t)=\left(\begin{array}{c}
p(t) f\left(t, x_{n}(t), y_{n}(t)\right) \\
q(t) g\left(t, x_{n}(t), y_{n}(t)\right) \\
\int_{0}^{1} \varphi(t) G\left(t, x_{n}(t), y_{n}(t)\right) d t \\
\int_{0}^{1} \psi(t) H\left(t, x_{n}(t), y_{n}(t)\right) d t \\
I\left(t_{1}, x_{n}\left(t_{1}\right), y_{n}\left(t_{1}\right)\right) \\
J\left(t_{1}, x_{n}\left(t_{1}\right), y_{n}\left(t_{1}\right)\right)
\end{array}\right)^{T} \quad \text { for }(x, y) \in E
$$

Since $f, g, G, H, I, J$ are Caratheodory functions, we know that $f\left(t,\left(t-t_{i}\right)^{\alpha-1} u,\left(t-t_{i}\right)^{\beta-1} v\right)$ is continuous on $\left[t_{i}, t_{i+1}\right] \times[-r, r]^{2}(i=0,1)$ respectively, so $f\left(t,\left(t-t_{i}\right)^{\alpha-1} u,\left(t-t_{i}\right)^{\beta-1} v\right)$ is uniformly continuous on $\left[t_{i}, t_{i+1}\right] \times[-r, r]^{2}(i=0,1)$ respectively.

Similarly, $g, G, H, I, J$ are uniformly continuous on $\left[t_{i}, t_{i+1}\right] \times[-r, r]^{2}(i=0,1)$ respectively. For any $\varepsilon>0$, there exists $\delta>0$ such that

$$
\begin{aligned}
& \left|f\left(t,\left(t-t_{k}\right)^{\alpha-1} u_{1},\left(t-t_{k}\right)^{\beta-1} v_{1}\right)-f\left(t,\left(t-t_{k}\right)^{\alpha-1} u_{2},\left(t-t_{k}\right)^{\beta-1} v_{2}\right)\right|<\varepsilon, \\
& \left|g\left(t,\left(t-t_{k}\right)^{\alpha-1} u_{1},\left(t-t_{k}\right)^{\beta-1} v_{1}\right)-g\left(t,\left(t-t_{k}\right)^{\alpha-1} u_{2},\left(t-t_{k}\right)^{\beta-1} v_{2}\right)\right|<\varepsilon, \\
& \left|G\left(t,\left(t-t_{k}\right)^{\alpha-1} u_{1},\left(t-t_{k}\right)^{\beta-1} v_{1}\right)-G\left(t,\left(t-t_{k}\right)^{\alpha-1} u_{2},\left(t-t_{k}\right)^{\beta-1} v_{2}\right)\right|<\varepsilon, \\
& \left|H\left(t,\left(t-t_{k}\right)^{\alpha-1} u_{1},\left(t-t_{k}\right)^{\beta-1} v_{1}\right)-H\left(t,\left(t-t_{k}\right)^{\alpha-1} u_{2},\left(t-t_{k}\right)^{\beta-1} v_{2}\right)\right|<\varepsilon
\end{aligned}
$$

hold for all $t \in\left(t_{k}, t_{k+1}\right](k=0,1)$ and

$$
\begin{aligned}
& \left|I\left(t_{1},\left(1-t_{1}\right)^{\alpha-1} u_{1},\left(1-t_{1}\right)^{\beta-1} v_{1}\right)-I\left(t_{1},\left(1-t_{1}\right)^{\alpha-1} u_{2},\left(1-t_{1}\right)^{\beta-1} v_{2}\right)\right|<\varepsilon \\
& \left|J\left(t_{1},\left(1-t_{1}\right)^{\alpha-1} u_{1},\left(1-t_{1}\right)^{\beta-1} v_{1}\right)-J\left(t_{1},\left(1-t_{1}\right)^{\alpha-1} u_{2},\left(1-t_{1}\right)^{\beta-1} v_{2}\right)\right|<\varepsilon
\end{aligned}
$$

for all $k=0,1,\left|u_{1}-u_{2}\right|<\delta$ and $\left|v_{1}-v_{2}\right|<\delta$ with $u_{1}, u_{2}, v_{1}, v_{2} \in[-, r, r]$.

From $\left(x_{n}, y_{n}\right) \rightarrow\left(x_{0}, y_{0}\right)$, there exists $N$ such that

$$
\begin{aligned}
& \left(t-t_{k}\right)^{1-\alpha}\left|x_{n}(t)-x_{0}(t)\right|<\delta, t \in\left(t_{k}, t_{k+1}\right], k=0,1, n>N \\
& \left(t-t_{k}\right)^{1-\beta}\left|y_{n}(t)-y_{0}(t)\right|<\delta, t \in\left(t_{k}, t_{k+1}\right], k=0,1, n>N .
\end{aligned}
$$


Hence, we get

$$
\begin{aligned}
& \int_{0}^{1}\left|p(t) f\left(t, x_{n}(t), y_{n}(t)\right)-p(t) f\left(t, x_{0}(t), y_{0}(t)\right)\right| d t \\
= & \sum_{k=0}^{1} \int_{t_{k}}^{t_{k+1}} \mid p(t) f\left(t,\left(t-t_{k}\right)^{\alpha-1}\left(t-t_{k}\right)^{1-\alpha} x_{n}(t),\left(t-t_{k}\right)^{\beta-1}\left(t-t_{k}\right)^{1-\beta} y_{n}(t)\right) \\
& -p(t)\left(t,\left(t-t_{k}\right)^{\alpha-1}\left(t-t_{k}\right)^{1-\alpha} x_{0}(t),\left(t-t_{k}\right)^{\beta-1}\left(t-t_{k}\right)^{1-\beta} y_{0}(t)\right) \mid d t \\
< & \sum_{k=0}^{1} \int_{t_{k}}^{t_{k+1}} \varepsilon p(t) d t=\varepsilon \int_{0}^{1} p(t) d t, n>N .
\end{aligned}
$$

It follows that, for all $n>N$, it holds that

$$
\left|\int_{0}^{1} p(t) f\left(t, x_{n}(t), y_{n}(t)\right) d t-\int_{0}^{1} p(t) f\left(t, x_{0}(t), y_{0}(t)\right) d t\right|<\varepsilon \int_{0}^{1} p(t) d t .
$$

Similarly for all $n>N$, we get

$$
\begin{aligned}
& \left|\int_{0}^{1} q(t) g\left(t, x_{n}(t), y_{n}(t)\right) d t-\int_{0}^{1} q(t) g\left(t, x_{0}(t), y_{0}(t)\right) d t\right|<\varepsilon \int_{0}^{1} q(t) d t \\
& \left|\int_{0}^{1} \varphi(t) G\left(t, x_{n}(t), y_{n}(t)\right) d t-\int_{0}^{1} \varphi(t) G\left(t, x_{0}(t), y_{0}(t)\right) d t\right|<\varepsilon \int_{0}^{1} \varphi(t) d t \\
& \left|\int_{0}^{1} \psi(t) H\left(t, x_{n}(t), y_{n}(t)\right) d t-\int_{0}^{1} \psi(t) H\left(t, x_{0}(t), y_{0}(t)\right) d t\right|<\varepsilon \int_{0}^{1} \psi(t) d t
\end{aligned}
$$

and

$$
\begin{aligned}
& \left|I\left(t_{1}, x_{n}\left(t_{1}\right), y_{n}\left(t_{1}\right)\right)-I\left(t_{1}, x_{0}\left(t_{1}\right), y_{0}\left(t_{1}\right)\right)\right|<\varepsilon \\
& \left|J\left(t_{1}, x_{n}\left(t_{1}\right), y_{n}\left(t_{1}\right)\right)-J\left(t_{1}, x_{0}\left(t_{1}\right), y_{0}\left(t_{1}\right)\right)\right|<\varepsilon .
\end{aligned}
$$

Then (16)-(21) imply that $\left\|N\left(x_{n}, y_{n}\right)-N\left(x_{0}, y_{0}\right)\right\| \rightarrow 0, n \rightarrow \infty$. It follows that $N$ is continuous. Let $P: X \rightarrow X$ and $Q: Y \rightarrow Y$ be defined by (11) and (13). For $(u, v, a, b, c, d) \in \operatorname{Im} L=Z$, let

$$
K_{P}(u, v, a, b, c, d)(t)=(x(t), y(t)),
$$

where $x(t)$ and $y(t)$ are defined by (9) and (10) respectively.

One can sow that $K_{P}(u, v, a, b, c, d) \in D(L) \bigcap E$ and $K_{P}: \operatorname{Im} L \rightarrow D(L) \cap \operatorname{Ker} P$ is the inverse of $L: D(L) \bigcap \operatorname{Ker} P \rightarrow \operatorname{Im} L$. The isomorphism $\wedge: \operatorname{Ker} L \rightarrow Y / \operatorname{Im} L$ is given by

$$
\wedge(0,0)=(0,0,0,0,0,0) .
$$

Furthermore, one has

$$
Q N(x, y)(t)=(0,0,0,0,0, \cdots, 0,0 \cdots, 0)
$$

and

$$
K_{p}(I-Q) N(x, y)(t)=K_{p} N(x, y)(t)=\left(x_{1}(t), y_{1}(t)\right)
$$


where

$$
\begin{aligned}
& \left\{\begin{array}{l}
I\left(t_{1}, x\left(t_{1}\right), y\left(t_{1}\right)\right) \Gamma(\alpha)^{2}\left(1-t_{1}\right)^{\alpha-1} E_{\alpha, \alpha}\left(\lambda\left(1-t_{1}\right)^{\alpha}\right) t^{\alpha-1} E_{\alpha, \alpha}\left(\lambda t^{\alpha}\right) \\
-\Gamma(\alpha) t^{\alpha-1} E_{\alpha, \alpha}\left(\lambda t^{\alpha}\right) \int_{0}^{1} \varphi(s) G(s, x(s), y(s)) d s
\end{array}\right. \\
& x_{1}(t)=\left\{\begin{array}{l}
+\Gamma(\alpha) t^{\alpha-1} E_{\alpha, \alpha}\left(\lambda t^{\alpha}\right) \int_{t_{1}}^{1}(1-s)^{\alpha-1} E_{\alpha, \alpha}\left(\lambda(1-s)^{\alpha}\right) p(s) f(s, x(s), y(s)) d s \\
+\int_{0}^{t}(t-s)^{\alpha-1} E_{\alpha, \alpha}\left(\lambda(t-s)^{\alpha}\right) p(s) f(s, x(s), y(s)) d s, \quad t \in\left(0, t_{1}\right],
\end{array}\right. \\
& I\left(t_{1}, x\left(t_{1}\right), y\left(t_{1}\right)\right) \Gamma(\alpha)\left(t-t_{1}\right)^{\alpha-1} E_{\alpha, \alpha}\left(\lambda\left(t-t_{1}\right)^{\alpha}\right) \\
& +\int_{t_{1}}^{t}(t-s)^{\alpha-1} E_{\alpha, \alpha}\left(\lambda(t-s)^{\alpha}\right) p(s) f(s, x(s), y(s)) d s, \quad t \in\left(t_{1}, 1\right] .
\end{aligned}
$$

and

$$
y_{1}(t)= \begin{cases}J\left(t_{1}, x\left(t_{1}\right), y\left(t_{1}\right)\right) \Gamma(\beta)^{2}\left(1-t_{1}\right)^{\beta-1} E_{\beta, \beta}\left(\mu\left(1-t_{1}\right)^{\beta}\right) t^{\beta-1} E_{\beta, \beta}\left(\mu t^{\beta}\right) \\ -\Gamma(\beta) t^{\beta-1} E_{\beta, \beta}\left(\mu t^{\beta}\right) \int_{0}^{1} \varphi(s) H(s, x(s), y(s)) d s \\ +\Gamma(\beta) t^{\beta-1} E_{\beta, \beta}\left(\mu t^{\beta}\right) \int_{t_{1}}^{1}(1-s)^{\beta-1} E_{\beta, \beta}\left(\mu(1-s)^{\beta}\right) q(s) g(s, x(s), y(s)) d s \\ +\int_{0}^{t}(t-s)^{\beta-1} E_{\beta, \beta}\left(\mu(t-s)^{\beta}\right) q(s) g(s, x(s), y(s)) d s, \quad t \in\left(0, t_{1}\right], \\ J\left(t_{1}, x\left(t_{1}\right), y\left(t_{1}\right)\right) \Gamma(\beta)\left(t-t_{1}\right)^{\beta-1} E_{\beta, \beta}\left(\mu\left(t-t_{1}\right)^{\beta}\right) \\ +\int_{t_{1}}^{t}(t-s)^{\beta-1} E_{\beta, \beta}\left(\mu(t-s)^{\beta}\right) q(s) g(s, x(s), y(s)) d s, \quad t \in\left(t_{1}, 1\right] .\end{cases}
$$

Let $\Omega$ be a bounded open subset of $E$ satisfying $\bar{\Omega} \bigcap D(L) \neq \varnothing$. We have $\|(x, y)\| \leq r<+\infty$ for all $(x, y) \in \Omega$. Since $f$ is a Caratheodory function, then $f\left(t,\left(t-t_{k}\right)^{\alpha-1} x,\left(t-t_{k}\right)^{\beta-1} y\right)$ is continuous both on $\left(t_{k}, t_{k+1}\right] \times[-r, r]^{2}(k=0,1)$ and there exist the limits

$$
\lim _{t \rightarrow 0^{+}} f\left(t, t^{\alpha-1} x, t^{\beta-1} y\right), \lim _{t \rightarrow t_{1}^{+}} f\left(t,\left(t-t_{1}\right)^{\alpha-1} x,\left(t-t_{1}\right)^{\beta-1} y\right)
$$

for every $(x, y) \in[-r, r]^{2}$. Then $f\left(t,\left(t-t_{!}\right)^{\alpha-1} x,\left(t-t_{1}\right)^{\beta-1} y\right)$ is bounded on $\left[t_{k}, t_{k+1}\right] \times[-r, r]^{2}(k=$ $0,1)$.

Similarly, $f, g, G, H, I, J$ are Caratheodory functions, there exists a constant $M>0$ such 
that

$$
\begin{gathered}
|f(t, x(t), y(t))|=\left|f\left(t,\left(t-t_{k}\right)^{\alpha-1}\left(t-t_{k}\right)^{1-\alpha} x(t),\left(t-t_{k}\right)^{\beta-1}\left(t-t_{k}\right)^{1-\beta} y(t)\right)\right| \leq M, \\
|g(t, x(t), y(t))| \leq M, \\
|G(t, x(t), y(t))| \leq M,
\end{gathered}
$$

and $|H(t, x(t), y(t))| \leq M$, hold for all $t \in(0,1)$

$$
\begin{gathered}
\left|I\left(t_{1}, x\left(t_{1}\right), y\left(t_{1}\right)\right)\right| \leq M, \\
\left|J\left(t_{1}, x\left(t_{1}\right), y\left(t_{1}\right)\right)\right| \leq M .
\end{gathered}
$$

Step (ii) Prove that $Q N(\bar{\Omega})$ is bounded.

It follows from (23) that $Q N(\bar{\Omega})$ is bounded.

Step (iii) Prove that $K_{P}(I-Q) N: \bar{\Omega} \rightarrow E$ is compact, i.e., prove that $K_{P}(I-Q) N(\bar{\Omega})$ is relatively compact. This is divided into three sub-steps:

Sub-step (iii1) Prove that $K_{P}(I-Q) N(\bar{\Omega})$ is uniformly bounded.

Using (26). We have for $t \in\left(0, t_{1}\right]$ that

$$
\begin{aligned}
& t^{1-\alpha}\left|x_{1}(t)\right| \leq \mid I\left(t_{1}, x\left(t_{1}, y\left(t_{1}\right)\right) \mid \Gamma(\alpha)^{2}\left(1-t_{1}\right)^{\alpha-1} E_{\alpha, \alpha}\left(\lambda\left(1-t_{1}\right)^{\alpha}\right) E_{\alpha, \alpha}\left(\lambda t^{\alpha}\right)\right. \\
& +\Gamma(\alpha) E_{\alpha, \alpha}\left(\lambda t^{\alpha}\right) \int_{0}^{1}|\varphi(s) G(s, x(s), y(s))| d s \\
& +\Gamma(\alpha) E_{\alpha, \alpha}\left(\lambda t^{\alpha}\right) \int_{t_{1}}^{1}(1-s)^{\alpha-1} E_{\alpha, \alpha}\left(\lambda(1-s)^{\alpha}\right)|p(s) f(s, x(s), y(s))| d s \\
& +t^{1-\alpha} \int_{0}^{t}(t-s)^{\alpha-1} E_{\alpha, \alpha}\left(\lambda(t-s)^{\alpha}\right)|p(s) f(s, x(s), y(s))| d s \\
& \leq M \Gamma(\alpha)^{2}\left(1-t_{1}\right)^{\alpha-1} E_{\alpha, \alpha}\left(\lambda\left(1-t_{1}\right)^{\alpha}\right) E_{\alpha, \alpha}(|\lambda|)+M \Gamma(\alpha) E_{\alpha, \alpha}(|\lambda|)\|\varphi\|_{1} \\
& +M \Gamma(\alpha) E_{\alpha, \alpha}(|\lambda|) \int_{t_{1}}^{1}(1-s)^{\alpha-1} E_{\alpha, \alpha}\left(\lambda(1-s)^{\alpha}\right)\left(s-t_{1}\right)^{k_{1}}(1-s)^{l_{1}} d s \\
& +M t^{1-\alpha} \int_{0}^{t}(t-s)^{\alpha-1} E_{\alpha, \alpha}\left(\lambda(t-s)^{\alpha}\right) s^{k_{1}}\left(t_{1}-s\right)^{l_{1}} d s \\
& \leq M \Gamma(\alpha)^{2}\left(1-t_{1}\right)^{\alpha-1} E_{\alpha, \alpha}\left(\lambda\left(1-t_{1}\right)^{\alpha}\right) E_{\alpha, \alpha}(|\lambda|)+M \Gamma(\alpha) E_{\alpha, \alpha}(|\lambda|)\|\varphi\|_{1} \\
& +M \Gamma(\alpha) E_{\alpha, \alpha}(|\lambda|) \sum_{j=0}^{+\infty} \int_{t_{1}}^{1}(1-s)^{\alpha+l_{1}-1} \frac{\lambda^{j}(1-s)^{\alpha j}}{\Gamma((j+1) \alpha)}\left(s-t_{1}\right)^{k_{1}} d s \\
& +M t^{1-\alpha} \sum_{j=0}^{+\infty} \int_{0}^{t}(t-s)^{\alpha-1} \frac{\lambda^{j}(t-s)^{\alpha j}}{\Gamma((j+1) \alpha)} s^{k_{1}}(t-s)^{l_{1}} d s
\end{aligned}
$$




$$
\begin{aligned}
& \leq M \Gamma(\alpha)^{2}\left(1-t_{1}\right)^{\alpha-1} E_{\alpha, \alpha}\left(\lambda\left(1-t_{1}\right)^{\alpha}\right) E_{\alpha, \alpha}(|\lambda|)+M \Gamma(\alpha) E_{\alpha, \alpha}(|\lambda|)\|\varphi\|_{1} \\
& +M \Gamma(\alpha) E_{\alpha, \alpha}(|\lambda|) \sum_{j=0}^{+\infty} \frac{\lambda^{j}}{\Gamma((j+1) \alpha)} \int_{t_{1}}^{1}(1-s)^{\alpha+\alpha j+l_{1}-1}\left(s-t_{1}\right)^{k_{1}} d s \\
& +M t^{1-\alpha} \sum_{j=0}^{+\infty} \frac{\lambda^{j}}{\Gamma((j+1) \alpha)} \int_{0}^{t}(t-s)^{\alpha+\alpha j+l_{1}-1} s^{k_{1}} d s \\
& =M \Gamma(\alpha)^{2}\left(1-t_{1}\right)^{\alpha-1} E_{\alpha, \alpha}\left(\lambda\left(1-t_{1}\right)^{\alpha}\right) E_{\alpha, \alpha}(|\lambda|)+M \Gamma(\alpha) E_{\alpha, \alpha}(|\lambda|)\|\varphi\|_{1} \\
& +M \Gamma(\alpha) E_{\alpha, \alpha}(|\lambda|) \sum_{j=0}^{+\infty} \frac{\lambda^{j} t^{\alpha+\alpha j+k_{1}+l_{1}}}{\Gamma((j+1) \alpha)} \mathbf{B}\left(\alpha+\alpha j+l_{1}, k_{1}+1\right) \\
& +M \sum_{j=0}^{+\infty} \frac{\lambda^{j} t^{1+\alpha j+l_{1}+k_{1}}}{\Gamma((j+1) \alpha)} \mathbf{B}\left(\alpha+\alpha j+l_{1}, k_{1}+1\right) \\
& \leq M \Gamma(\alpha)^{2}\left(1-t_{1}\right)^{\alpha-1} E_{\alpha, \alpha}\left(\lambda\left(1-t_{1}\right)^{\alpha}\right) E_{\alpha, \alpha}(|\lambda|)+M \Gamma(\alpha) E_{\alpha, \alpha}(|\lambda|)\|\varphi\|_{1} \\
& +M \Gamma(\alpha) E_{\alpha, \alpha}(|\lambda|) \sum_{j=0}^{+\infty} \frac{\lambda^{j} t^{\alpha j}}{\Gamma((j+1) \alpha)} \mathbf{B}\left(\alpha+l_{1}, k_{1}+1\right)+M \sum_{j=0}^{+\infty} \frac{\lambda^{j} t^{\alpha j}}{\Gamma((j+1) \alpha)} \mathbf{B}\left(\alpha+l_{1}, k_{1}+1\right) \\
& \leq M \Gamma(\alpha)^{2}\left(1-t_{1}\right)^{\alpha-1} E_{\alpha, \alpha}\left(\lambda\left(1-t_{1}\right)^{\alpha}\right) E_{\alpha, \alpha}(|\lambda|)+M \Gamma(\alpha) E_{\alpha, \alpha}(|\lambda|)\|\varphi\|_{1} \\
& +M \Gamma(\alpha) E_{\alpha, \alpha}(|\lambda|) E_{\alpha, \alpha}(|\lambda|) \mathbf{B}\left(\alpha+l_{1}, k_{1}+1\right)+M E_{\alpha, \alpha}(|\lambda|) \mathbf{B}\left(\alpha+l_{1}, k_{1}+1\right)<+\infty .
\end{aligned}
$$

For $t \in\left(t_{1}, 1\right]$, we have

$$
\begin{aligned}
& \left(t-t_{1}\right)^{1-\alpha}\left|x_{1}(t)\right| \leq \mid I\left(t_{1}, x\left(t_{1}, y\left(t_{1}\right)\right) \mid \Gamma(\alpha) E_{\alpha, \alpha}\left(\lambda\left(t-t_{1}\right)^{\alpha}\right)\right. \\
& +\left(t-t_{1}\right)^{1-\alpha} \int_{t_{1}}^{t}(t-s)^{\alpha-1} E_{\alpha, \alpha}\left(\lambda(t-s)^{\alpha}\right)|p(s) f(s, x(s), y(s))| d s \\
& \leq M \Gamma(\alpha) E_{\alpha, \alpha}(|\lambda|)+M\left(t-t_{1}\right)^{1-\alpha} \int_{t_{1}}^{t}(t-s)^{\alpha-1} E_{\alpha, \alpha}\left(\lambda(t-s)^{\alpha}\right)\left(s-t_{1}\right)^{k_{1}}(1-s)^{l_{1}} d s \\
& =M \Gamma(\alpha) E_{\alpha, \alpha}(|\lambda|)+M\left(t-t_{1}\right)^{1-\alpha} \sum_{j=0}^{\infty} \frac{\lambda^{j}}{\Gamma(\alpha(j+1))} \int_{t_{1}}^{t}(t-s)^{\alpha+\alpha j+l_{1}-1}\left(s-t_{1}\right)^{k_{1}} d s \\
& \leq M \Gamma(\alpha) E_{\alpha, \alpha}(|\lambda|)+M \sum_{j=0}^{\infty} \frac{\lambda^{j}\left(t-t_{1}\right)^{1+\alpha j+k_{1}+l_{1}}}{\Gamma(\alpha(j+1))} \mathbf{B}\left(\alpha+\alpha j+l_{1}, k_{1}+1\right) \\
& \leq M \Gamma(\alpha) E_{\alpha, \alpha}(|\lambda|)+M \sum_{j=0}^{\infty} \frac{\lambda^{j}\left(t-t_{1}\right)^{\alpha j}}{\Gamma(\alpha(j+1))} \mathbf{B}\left(\alpha+l_{1}, k_{1}+1\right) \\
& =M \Gamma(\alpha) E_{\alpha, \alpha}(|\lambda|)+M E_{\alpha, \alpha}(|\lambda|) \mathbf{B}\left(\alpha+l_{1}, k_{1}+1\right)<+\infty .
\end{aligned}
$$

From above discussion, there exists $M_{1}>0$ such that $\left\|x_{1}\right\|_{\infty} \leq M_{1}<+\infty$. Similarly, we can show 
that there exist $M_{2}>0$ such that $\left\|y_{1}\right\|_{\infty} \leq M_{2}<+\infty$. Hence $K_{P}(I-Q) N(\bar{\Omega})$ is uniformly bounded.

Sub-step (iii2) Prove that $K_{P}(I-Q) N(\bar{\Omega})$ is equi-continuous on each subinterval $[e, f]$ both $\subseteq\left(0, t_{1}\right]$ and $\left(t_{1}, 1\right]$, respectively.

Note $\left(K_{P}(I-Q) N(x, y)=\left(x_{1}, y_{1}\right), x_{1}, y_{1}\right.$ are defined by (24) and (25). Define

$$
\left(t-t_{i}\right)^{1-\alpha} x_{1}(t)=\left\{\begin{array}{c}
\left(t-t_{i}\right)^{1-\alpha} x_{t}(t), t \in\left(t_{i}, t_{i+1}\right] \\
\lim _{t \rightarrow t_{i}^{+}}\left(t-t_{i}\right)^{1-\alpha} x_{1}(t), t=t_{i}
\end{array}\right.
$$

Then $\left(t-t_{i}\right)^{1-\alpha} x_{1}(t)$ is continuous on $\left[t_{i}, t_{i+1}\right]$. So $\left\{t \rightarrow\left(t-t_{i}\right)^{1-\alpha} x_{1}(t):(x, y) \in \bar{\Omega}\right\}$ is equi-continuous on $\left[t_{i}, t_{i+1}\right]$. Hence $\left\{t \rightarrow\left(t-t_{i}\right)^{1-\alpha} x_{1}(t):(x, y) \in \bar{\Omega}\right\}$ is equi-continuous on $\left(t_{i}, t_{i+1}\right](i=0,1)$. Similarly we can show that $\left\{t \rightarrow\left(t-t_{i}\right)^{1-\beta} y_{1}(t):(x, y) \in \bar{\Omega}\right\}$ is equi-continuous on $\left(t_{i}, t_{i+1}\right](i=0,1)$. So $K_{P}(I-Q) N(\bar{\Omega})$ is relatively compact. Then $N$ is $L$-compact. The proofs are completed.

\section{Main Results}

Now, we prove that main theorem in this paper. We need the following assumptions:

(C) $\Phi$ is a sup-multiplicative-like function with its supporting function $w$, the inverse function of $\Phi$ is $\Phi^{-1}$ with supporting function $\nu$.

(D) $f, g, H, G, I, J$ are Caratheodory functions and satisfy that there exist nonnegative constants $c_{i}, b_{i}, a_{i}(i=1,2), C_{i}, B_{i}, A_{i}(i=1,2)$ and $\bar{C}_{i}, \bar{B}_{i}, \bar{A}_{i}(i=1,2)$ such that

$$
\begin{aligned}
& \left|f\left(t,\left(t-t_{k}\right)^{\alpha-1} x,\left(t-t_{k}\right)^{\beta-1} y\right)\right| \leq c_{1}+b_{1}|x|+a_{1} \Phi^{-1}(|y|), t \in\left(t_{k}, t_{k+1}\right], k=0,1, \\
& \left|g\left(t,\left(t-t_{k}\right)^{\alpha-1} x,\left(t-t_{k}\right)^{\beta-1} y\right)\right| \leq c_{2}+b_{2} \Phi(|x|)+a_{2}|y|, t \in\left(t_{k}, t_{k+1}\right], k=0,1 \\
& \left|G\left(t,\left(t-t_{k}\right)^{\alpha-1} x,\left(t-t_{k}\right)^{\beta-1} y\right)\right| \leq C_{1}+B_{1}|x|+A_{1} \Phi^{-1}(|y|), t \in\left(t_{k}, t_{k+1}\right], k=0,1 \\
& \left|H\left(t,\left(t-t_{k}\right)^{\alpha-1} x,\left(t-t_{k}\right)^{\beta-1} y\right)\right| \leq C_{2}+B_{2} \Phi(|x|)+A_{2}|y|, t \in\left(t_{k}, t_{k+1}\right], k=0,1 \\
& \left|I\left(t_{1},\left(1-t_{1}\right)^{\alpha-1} x,\left(1-t_{1}\right)^{\beta-1} y\right)\right| \leq \bar{C}_{1}+\bar{B}_{1}|x|+\bar{A}_{1} \Phi^{-1}(|y|) \\
& \left|J\left(t_{1},\left(1-t_{1}\right)^{\alpha-1} x,\left(1-t_{1}\right)^{\beta-1} y\right)\right| \leq \bar{C}_{2}+\bar{B}_{2} \Phi(|x|)+\bar{A}_{2}|y|
\end{aligned}
$$

Denote

$$
\begin{aligned}
\mu_{1}= & : \max \left\{\Gamma(\alpha)^{2} E_{\alpha, \alpha}(|\lambda|)^{2} \bar{C}_{1}+\Gamma(\alpha) E_{\alpha, \alpha}(|\lambda|)|| \varphi \|_{1} C_{1}\right. \\
& +\Gamma(\alpha) E_{\alpha, \alpha}(|\lambda|)^{2} \mathbf{B}\left(\alpha+l_{1}, k_{1}+1\right) c_{1}+E_{\alpha, \alpha}(|\lambda|) \mathbf{B}\left(\alpha+l_{1}, k_{1}+1\right) c_{1} \\
& \left.\Gamma(\alpha) E_{\alpha, \alpha}(|\lambda|) \bar{C}_{1}+E_{\alpha, \alpha}(|\lambda|) \mathbf{B}\left(\alpha+l_{1}, k_{1}+1\right) c_{1}\right\}
\end{aligned}
$$




$$
\begin{aligned}
\sigma_{1}=: & \max \left\{\Gamma(\alpha)^{2} E_{\alpha, \alpha}(|\lambda|)^{2} \bar{B}_{1}+\Gamma(\alpha) E_{\alpha, \alpha}(|\lambda|)\|\varphi\|_{1} B_{1}\right. \\
& +\Gamma(\alpha) E_{\alpha, \alpha}(|\lambda|)^{2} \mathbf{B}\left(\alpha+l_{1}, k_{1}+1\right) b_{1}+E_{\alpha, \alpha}(|\lambda|) \mathbf{B}\left(\alpha+l_{1}, k_{1}+1\right) b_{1}, \\
& \left.\Gamma(\alpha) E_{\alpha, \alpha}(|\lambda|) \bar{B}_{1}+E_{\alpha, \alpha}(|\lambda|) \mathbf{B}\left(\alpha+l_{1}, k_{1}+1\right) b_{1}\right\}, \\
\delta_{1}=: & \max \left\{\Gamma(\alpha)^{2} E_{\alpha, \alpha}(|\lambda|)^{2} \bar{A}_{1}+\Gamma(\alpha) E_{\alpha, \alpha}(|\lambda|)\|\varphi\|_{1} A_{1}\right. \\
& +\Gamma(\alpha) E_{\alpha, \alpha}(|\lambda|)^{2} \mathbf{B}\left(\alpha+l_{1}, k_{1}+1\right) a_{1}+E_{\alpha, \alpha}(|\lambda|) \mathbf{B}\left(\alpha+l_{1}, k_{1}+1\right) a_{1}, \\
& \left.\Gamma(\alpha) E_{\alpha, \alpha}(|\lambda|) \bar{A}_{1}+E_{\alpha, \alpha}(|\lambda|) \mathbf{B}\left(\alpha+l_{1}, k_{1}+1\right) a_{1}\right\},
\end{aligned}
$$

and

$$
\begin{aligned}
\mu_{2}= & \max \left\{\Gamma(\beta)^{2} E_{\beta, \beta}(|\mu|)^{2} \bar{C}_{2}+\Gamma(\beta) E_{\beta, \beta}(|\mu|)|| \psi \|_{1} C_{2}\right. \\
& +\Gamma(\beta) E_{\beta, \beta}(|\mu|)^{2} \mathbf{B}\left(\beta+l_{2}, k_{2}+1\right) c_{2}+E_{\beta, \beta}(|\mu|) \mathbf{B}\left(\beta+l_{2}, k_{2}+1\right) c_{2}, \\
& \left.\Gamma(\beta) E_{\beta, \beta}(|\mu|) \bar{C}_{2}+E_{\beta, \beta}(|\mu|) \mathbf{B}\left(\beta+l_{2}, k_{2}+1\right) c_{2}\right\}, \\
\sigma_{2}=: & \max \left\{\Gamma(\beta)^{2} E_{\beta, \beta}(|\mu|)^{2} \bar{B}_{2}+\Gamma(\beta) E_{\beta, \beta}(|\mu|)|| \psi \|_{1} B_{2}\right. \\
& +\Gamma(\beta) E_{\beta, \beta}(|\mu|)^{2} \mathbf{B}\left(\beta+l_{2}, k_{2}+1\right) b_{2}+E_{\beta, \beta}(|\mu|) \mathbf{B}\left(\beta+l_{2}, k_{2}+1\right) b_{2}, \\
& \left.\Gamma(\beta) E_{\beta, \beta}(|\mu|) \bar{B}_{2}+E_{\beta, \beta}(|\mu|) \mathbf{B}\left(\beta+l_{2}, k_{2}+1\right) b_{2}\right\}, \\
\delta_{2}=: & \max \left\{\Gamma(\beta)^{2} E_{\beta, \beta}(|\mu|)^{2} \bar{A}_{2}+\Gamma(\beta) E_{\beta, \beta}(|\mu|)\|\psi\|_{1} A_{2}\right. \\
& +\Gamma(\beta) E_{\beta, \beta}(|\mu|)^{2} \mathbf{B}\left(\beta+l_{2}, k_{2}+1\right) a_{2}+E_{\beta, \beta}(|\mu|) \mathbf{B}\left(\beta+l_{2}, k_{2}+1\right) a_{2}, \\
& \left.\Gamma(\beta) E_{\beta, \beta}(|\mu|) \bar{A}_{2}+E_{\beta, \beta}(|\mu|) \mathbf{B}\left(\beta+l_{2}, k_{2}+1\right) a_{2}\right\} .
\end{aligned}
$$

Theorem 3.1. Suppose that $(\mathrm{C})$ and $(\mathrm{D})$ hold. Then BVP(4) has at least one solution if

$$
\begin{array}{ll}
\sigma_{1}<1, & \frac{\sigma_{2}}{w\left(\left(1-\delta_{1}\right) /\left(2 \delta_{1}\right)\right)}+\delta_{2}<1 \\
\text { or } & \\
\delta_{2}<1, & \sigma_{1}+\delta_{1} \nu\left(\frac{2 \sigma_{2}}{1-\delta_{2}}\right)<1 .
\end{array}
$$

Proof: To apply Lemma 2.1, we should define an open bounded subset $\Omega$ of $E$ centered at zero such that assumptions in Lemma 2.1 hold. To obtain $\Omega$.

Let $\Omega_{1}=\{(x, y) \in E \cap D(L) \backslash \operatorname{Ker} L, L(x, y)=\theta N(x, y)$ for some $\theta \in(0,1)\}$. We prove that $\Omega_{1}$ is bounded. 
For $(x, y) \in \Omega_{1}$, we get $L(x, y)=\theta N(x, y)$ and $N(x, y) \in \operatorname{Im} L$. Then

$$
\left\{\begin{array}{l}
D_{t_{k}^{+}}^{\alpha} x(t)-\lambda x(t)=\theta p(t) f(t, x(t), y(t)), \quad t \in\left(t_{k}, t_{k+1}\right], k=0,1 \\
D_{t_{k}^{+}}^{\beta} y(t)-\mu y(t)=\theta q(t) g(t, x(t), y(t)), \quad t \in\left(t_{k}, t_{k+1}\right], k=0,1 \\
x(1)-\lim _{t \rightarrow 0} t^{1-\alpha} x(t)=\theta \int_{0}^{1} \varphi(s) G(s, x(s), y(s)) d s \\
y(1)-\lim _{t \rightarrow 0} t^{1-\beta} y(t)=\theta \int_{0}^{1} \psi(s) H(s, x(s), y(s)) d s \\
\lim _{t \rightarrow t_{1}^{+}}\left[t-t_{1}\right]^{1-\alpha} x(t)=\theta I\left(t_{1}, x\left(t_{1}\right), y\left(t_{1}\right)\right) \\
\lim _{t \rightarrow t_{1}^{+}}\left[t-t_{1}\right]^{1-\beta} y(t)=\theta J\left(t_{1}, u\left(t_{1}\right), D_{0^{+}}^{\alpha} u\left(t_{1}\right)\right)
\end{array}\right.
$$

So

$$
x(t)= \begin{cases}\theta\left[I\left(t_{1}, x\left(t_{1}\right), y\left(t_{1}\right)\right) \Gamma(\alpha)^{2}\left(1-t_{1}\right)^{\alpha-1} E_{\alpha, \alpha}\left(\lambda\left(1-t_{1}\right)^{\alpha}\right) t^{\alpha-1} E_{\alpha, \alpha}\left(\lambda t^{\alpha}\right)\right. \\ -\Gamma(\alpha) t^{\alpha-1} E_{\alpha, \alpha}\left(\lambda t^{\alpha}\right) \int_{0}^{1} \varphi(s) G(s, x(s), y(s)) d s \\ +\Gamma(\alpha) t^{\alpha-1} E_{\alpha, \alpha}\left(\lambda t^{\alpha}\right) \int_{t_{1}}^{1}(1-s)^{\alpha-1} E_{\alpha, \alpha}\left(\lambda(1-s)^{\alpha}\right) p(s) f(s, x(s), y(s)) d s \\ \left.+\int_{0}^{t}(t-s)^{\alpha-1} E_{\alpha, \alpha}\left(\lambda(t-s)^{\alpha}\right) p(s) f(s, x(s), y(s)) d s\right], & t \in\left(0, t_{1}\right], \\ \theta\left[I\left(t_{1}, x\left(t_{1}\right), y\left(t_{1}\right)\right) \Gamma(\alpha)\left(t-t_{1}\right)^{\alpha-1} E_{\alpha, \alpha}\left(\lambda\left(t-t_{1}\right)^{\alpha}\right)\right. & \\ \left.+\int_{t_{1}}^{t}(t-s)^{\alpha-1} E_{\alpha, \alpha}\left(\lambda(t-s)^{\alpha}\right) p(s) f(s, x(s), y(s)) d s\right], & t \in\left(t_{1}, 1\right] .\end{cases}
$$

and

$$
y(t)= \begin{cases}\theta\left[J\left(t_{1}, x\left(t_{1}\right), y\left(t_{1}\right)\right) \Gamma(\beta)^{2}\left(1-t_{1}\right)^{\beta-1} E_{\beta, \beta}\left(\mu\left(1-t_{1}\right)^{\beta}\right) t^{\beta-1} E_{\beta, \beta}\left(\mu t^{\beta}\right)\right. \\ -\Gamma(\beta) t^{\beta-1} E_{\beta, \beta}\left(\mu t^{\beta}\right) \int_{0}^{1} \varphi(s) H(s, x(s), y(s)) d s \\ +\Gamma(\beta) t^{\beta-1} E_{\beta, \beta}\left(\mu t^{\beta}\right) \int_{t_{1}}^{1}(1-s)^{\beta-1} E_{\beta, \beta}\left(\mu(1-s)^{\beta}\right) q(s) g(s, x(s), y(s)) d s \\ \left.+\int_{0}^{t}(t-s)^{\beta-1} E_{\beta, \beta}\left(\mu(t-s)^{\beta}\right) q(s) g(s, x(s), y(s)) d s\right], & t \in\left(0, t_{1}\right], \\ \theta\left[J \left(t_{1}, x\left(t_{1}, y\left(t_{1}\right)\right) \Gamma(\beta)\left(t-t_{1}\right)^{\beta-1} E_{\beta, \beta}\left(\mu\left(t-t_{1}\right)^{\beta}\right)\right.\right. & \\ \left.+\int_{t_{1}}^{t}(t-s)^{\beta-1} E_{\beta, \beta}\left(\mu(t-s)^{\beta}\right) q(s) g(s, x(s), y(s)) d s\right], & t \in\left(t_{1}, 1\right] .\end{cases}
$$


Use (D), we get

$$
\begin{aligned}
& |f(t, x(t), y(t))|=f\left(t, t^{\alpha-1} t^{1-\alpha} x(t), t^{\beta-1} t^{1-\beta} y(t)\right) \mid \\
& \leq c_{1}+b_{1} t^{1-\alpha}|x(t)|+a_{1} \Phi^{-1}\left(t^{1-\beta}|y(t)|\right) \leq c_{1}+b_{1}\|x\|+a_{1} \Phi^{-1}(\|y\|), \\
& |G(t, x(t), y(t))| \leq C_{1}+B_{1}\|x\|+A_{1} \Phi^{-1}(\|y\|), \\
& \left|I\left(t_{1}, x\left(t_{1}\right), y\left(t_{1}\right)\right)\right| \leq \bar{C}_{1}+\bar{B}_{1}\|x\|+\bar{A}_{1} \Phi^{-1}(\|y\|) .
\end{aligned}
$$

Then, for $t \in\left(0, t_{1}\right]$, similarly to Sub-step (iii1), we have

$$
\begin{aligned}
& t^{1-\alpha}|x(t)| \leq\left|I\left(t_{1}, x\left(t_{1}\right), y\left(t_{1}\right)\right)\right| \Gamma(\alpha)^{2}\left(1-t_{1}\right)^{\alpha-1} E_{\alpha, \alpha}\left(\lambda\left(1-t_{1}\right)^{\alpha}\right) E_{\alpha, \alpha}\left(\lambda t^{\alpha}\right) \\
& +\Gamma(\alpha) E_{\alpha, \alpha}\left(\lambda t^{\alpha}\right) \int_{0}^{1}|\varphi(s) G(s, x(s), y(s))| d s \\
& +\Gamma(\alpha) E_{\alpha, \alpha}\left(\lambda t^{\alpha}\right) \int_{t_{1}}^{1}(1-s)^{\alpha-1} E_{\alpha, \alpha}\left(\lambda(1-s)^{\alpha}\right)|p(s) f(s, x(s), y(s))| d s \\
& +t^{1-\alpha} \int_{0}^{t}(t-s)^{\alpha-1} E_{\alpha, \alpha}\left(\lambda(t-s)^{\alpha}\right)|p(s) f(s, x(s), y(s))| d s \\
& \leq\left[\bar{C}_{1}+\bar{B}_{1}\|x\|+\bar{A}_{1} \Phi^{-1}(\|y\|)\right] \Gamma(\alpha)^{2}\left(1-t_{1}\right)^{\alpha-1} E_{\alpha, \alpha}\left(\lambda\left(1-t_{1}\right)^{\alpha}\right) E_{\alpha, \alpha}(|\lambda|) \\
& +\Gamma(\alpha) E_{\alpha, \alpha}(|\lambda|)\|\varphi\|_{1}\left[C_{1}+B_{1}\|x\|+A_{1} \Phi^{-1}(\|y\|)\right] \\
& +\Gamma(\alpha) E_{\alpha, \alpha}\left(\lambda t^{\alpha}\right) \int_{t_{1}}^{1}(1-s)^{\alpha-1} E_{\alpha, \alpha}\left(\lambda(1-s)^{\alpha}\right)\left(s-t_{1}\right)^{k_{1}}(1-s)^{l_{1}} d s\left[c_{1}+b_{1}\|x\|+a_{1} \Phi^{-1}(\|y\|)\right] \\
& +t^{1-\alpha} \int_{0}^{t}(t-s)^{\alpha-1} E_{\alpha, \alpha}\left(\lambda(t-s)^{\alpha}\right) s^{k_{1}}\left(t_{1}-s\right)^{l_{1}} d s\left[c_{1}+b_{1}\|x\|+a_{1} \Phi^{-1}(\|y\|)\right] \\
& \leq \Gamma(\alpha)^{2} E_{\alpha, \alpha}(|\lambda|)^{2} \bar{C}_{1}+\Gamma(\alpha) E_{\alpha, \alpha}(|\lambda|)\|\varphi\|_{1} C_{1} \\
& +\Gamma(\alpha) E_{\alpha, \alpha}(|\lambda|)^{2} \mathbf{B}\left(\alpha+l_{1}, k_{1}+1\right) c_{1}+E_{\alpha, \alpha}(|\lambda|) \mathbf{B}\left(\alpha+l_{1}, k_{1}+1\right) c_{1} \\
& +\left[\Gamma(\alpha)^{2} E_{\alpha, \alpha}(|\lambda|)^{2} \bar{B}_{1}+\Gamma(\alpha) E_{\alpha, \alpha}(|\lambda|)\|\varphi\|_{1} B_{1}\right. \\
& \left.+\Gamma(\alpha) E_{\alpha, \alpha}(|\lambda|)^{2} \mathbf{B}\left(\alpha+l_{1}, k_{1}+1\right) b_{1}+E_{\alpha, \alpha}(|\lambda|) \mathbf{B}\left(\alpha+l_{1}, k_{1}+1\right) b_{1}\right]\|x\| \\
& +\left[\Gamma(\alpha)^{2} E_{\alpha, \alpha}(|\lambda|)^{2} \bar{A}_{1}+\Gamma(\alpha) E_{\alpha, \alpha}(|\lambda|)\|\varphi\|_{1} A_{1}\right. \\
& \left.+\Gamma(\alpha) E_{\alpha, \alpha}(|\lambda|)^{2} \mathbf{B}\left(\alpha+l_{1}, k_{1}+1\right) a_{1}+E_{\alpha, \alpha}(|\lambda|) \mathbf{B}\left(\alpha+l_{1}, k_{1}+1\right) a_{1}\right] \Phi^{-1}(\|y\|) .
\end{aligned}
$$

For $t \in\left(t_{1}, 1\right]$, we have

$$
\begin{aligned}
& \left(t-t_{1}\right)^{1-\alpha}|x(t)| \leq\left|I\left(t_{1}, x\left(t_{1}\right), y\left(t_{1}\right)\right)\right| \Gamma(\alpha) E_{\alpha, \alpha}\left(\lambda\left(t-t_{1}\right)^{\alpha}\right) \\
& +\left(t-t_{1}\right)^{1-\alpha} \int_{t_{1}}^{t}(t-s)^{\alpha-1} E_{\alpha, \alpha}\left(\lambda(t-s)^{\alpha}\right)|p(s) f(s, x(s), y(s))| d s
\end{aligned}
$$




$$
\begin{aligned}
& \leq\left[\bar{C}_{1}+\bar{B}_{1}\|x\|+\bar{A}_{1} \Phi^{-1}(\|y\|)\right] \Gamma(\alpha) E_{\alpha, \alpha}(|\lambda|) \\
& +\left(t-t_{1}\right)^{1-\alpha} \int_{t_{1}}^{t}(t-s)^{\alpha-1} E_{\alpha, \alpha}\left(\lambda(t-s)^{\alpha}\right)\left(s-t_{1}\right)^{k_{1}}(1-s)^{l_{1}} d s\left[c_{1}+b_{1}\|x\|+a_{1} \Phi^{-1}(\|y\|)\right] \\
& \leq\left[\bar{C}_{1}+\bar{B}_{1}\|x\|+\bar{A}_{1} \Phi^{-1}(\|y\|)\right] \Gamma(\alpha) E_{\alpha, \alpha}(|\lambda|) \\
& +\left(t-t_{1}\right)^{1-\alpha} \int_{t_{1}}^{t}(t-s)^{\alpha-1} \sum_{k=0}^{\infty} \frac{\lambda^{k}(t-s)^{\alpha k}}{\alpha(k+1))}\left(s-t_{1}\right)^{k_{1}}(t-s)^{l_{1}} d s\left[c_{1}+b_{1}\|x\|+a_{1} \Phi^{-1}(\|y\|)\right] \\
& \leq \Gamma(\alpha) E_{\alpha, \alpha}(|\lambda|) \bar{C}_{1}+E_{\alpha, \alpha}(|\lambda|) \mathbf{B}\left(\alpha+l_{1}, k_{1}+1\right) c_{1} \\
& +\left[\Gamma(\alpha) E_{\alpha, \alpha}(|\lambda|) \bar{B}_{1}+E_{\alpha, \alpha}(|\lambda|) \mathbf{B}\left(\alpha+l_{1}, k_{1}+1\right) b_{1}\right]\|x\| \\
& \left.+\left[\Gamma(\alpha) E_{\alpha, \alpha}(|\lambda|) \bar{A}_{1}+E_{\alpha, \alpha}(|\lambda|) \mathbf{B}\left(\alpha+l_{1}, k_{1}+1\right) a_{1}\right] \Phi^{-1}(\|y\|)\right] .
\end{aligned}
$$

Then

$$
\|x\| \leq \mu_{1}+\sigma_{1}\|x\|+\delta_{1} \Phi^{-1}(\|y\|) .
$$

Similar to above discussion, we can prove that

$$
\|y\| \leq \mu_{2}+\sigma_{2} \Phi(\|x\|)+\delta_{2}\|y\| .
$$

Case 1. $\sigma_{1}<1, \quad \frac{\sigma_{2}}{w\left(\left(1-\delta_{1}\right) /\left(2 \delta_{1}\right)\right)}+\delta_{2}<1$.

From (31) we have Then (27) implies that $\|x\| \leq \frac{\mu_{1}}{1-\sigma_{1}}+\frac{\delta_{1}}{1-\sigma_{1}} \Phi^{-1}(\|y\|)$. Without loss of generality, suppose that $\|y\|>\Phi\left(\mu_{1} / \delta_{1}\right)$. Use (5), we get

$$
\begin{aligned}
& \|y\| \leq \mu_{2}+\sigma_{2} \Phi\left(\frac{\mu_{1}}{1-\sigma_{1}}+\frac{\delta_{1}}{1-\sigma_{1}} \Phi^{-1}(\|y\|)\right)+\delta_{2}\|y\| \\
& \leq \mu_{2}+\sigma_{2} \Phi\left(\frac{2 \delta_{1}}{1-\sigma_{1}} \Phi^{-1}(\|y\|)\right)+\delta_{2}\|y\| \\
& \leq \mu_{2}+\sigma_{2} \frac{\Phi\left(\Phi^{-1}(\|y\|)\right)}{w\left(\left(1-\delta_{1}\right) /\left(2 \delta_{1}\right)\right)}+\delta_{2}\|y\| \\
& =\mu_{2}+\sigma_{2} \frac{\|y\|}{w\left(\left(1-\delta_{1}\right) /\left(2 \delta_{1}\right)\right)}+\delta_{2}\|y\| .
\end{aligned}
$$

Form (27), there exists a constant $M_{1}>\Phi\left(\mu_{1} / \delta_{1}\right)$ such that $\|y\| \leq M_{1}$. Hence $\|x\| \leq \frac{\mu_{1}}{1-\sigma_{1}}+$ $\frac{\delta_{1}}{1-\sigma_{1}} \Phi^{-1}\left(M_{1}\right)$. It follows that $\Omega_{1}$ is bounded.

Case 2. $\delta_{2}<1, \quad \sigma_{1}+\delta_{1} \nu\left(\frac{2 \sigma_{2}}{1-\delta_{2}}\right)<1$.

From (32) we have $\|y\| \leq \frac{\mu_{2}}{1-\delta_{2}}+\frac{\sigma_{2}}{1-\delta_{2}} \Phi(\|x\|)$. Without loss of generality, suppose that $\|x\|>$ $\Phi^{-1}\left(\mu_{2} / \sigma_{2}\right)$. Use $(6)$, we get

$$
\begin{aligned}
& \|x\| \leq \mu_{1}+\sigma_{1}\|x\|+\delta_{1} \Phi^{-1}\left(\frac{\mu_{2}}{1-\delta_{2}}+\frac{\sigma_{2}}{1-\delta_{2}} \Phi(\|x\|)\right) \\
& \leq \mu_{1}+\sigma_{1}\|x\|+\delta_{1} \Phi^{-1}\left(\frac{2 \sigma_{2}}{1-\delta_{2}} \Phi(\|x\|)\right) \\
& \leq \mu_{1}+\sigma_{1}\|x\|+\delta_{1} \nu\left(\frac{2 \sigma_{2}}{1-\delta_{2}}\right)\|x\| .
\end{aligned}
$$


Form (27), there exists a constant $M_{1}>\Phi^{-1}\left(\mu_{2} / \sigma_{2}\right)$ such that $\|x\| \leq M_{1}$. Hence $\|y\| \leq \frac{\mu_{2}}{1-\delta_{2}}+$ $\frac{\sigma_{2}}{1-\delta_{2}} \Phi\left(M_{1}\right)$. It follows that $\Omega_{1}$ is bounded.

To apply Lemma 2.1, let $\Omega$ be a non-empty open bounded subset of $E$ such that $\Omega \supset \overline{\Omega_{1}}$ centered at zero.

It is easy to see from Lemma 2.2 that $L$ is a Fredholm operator of index zero and $N$ is $L$-compact on $\bar{\Omega}$. One can see that

$$
L(x, y) \neq \theta N(x, y) \text { for all }(x, y) \in E \cap \partial \Omega \text { and } \theta \in(0,1) .
$$

Thus, from Lemma 2.1,

$$
L(x, y)=N(x, y)
$$

has at least one solution $(x, y) \in E \cap \bar{\Omega}$. So $(x, y)$ is a solution of $\operatorname{BVP}(4)$. The proof of Theorem 3.1 is complete.

\section{An example}

Now, we present an example, which can not be covered by known results, to illustrate Theorem 2.1 .

Example 4.1. Consider the boundary value problem for fractional differential equation

$$
\left\{\begin{array}{l}
D_{*}^{\frac{2}{3}} x(t)-x(t)=t^{-\frac{1}{4}} f(t, x(t), y(t)), \quad t \in(0,1), t \neq \frac{1}{2}, \\
D_{*}^{\frac{1}{2}} y(t)-y(t)=t^{-\frac{1}{4}} g(t, x(t), y(t)), \quad t \in(0,1), t \neq \frac{1}{2}, \\
\lim _{t \rightarrow 1} t^{\frac{1}{3}} x(t)-\lim _{t \rightarrow 0} t^{\frac{1}{3}} x(t)=\frac{1}{2} \int_{0}^{1} s^{-\frac{1}{2}} G(s, x(s), y(s)) d s \\
\lim _{t \rightarrow 1^{2}} t^{\frac{1}{2}} y(t)-\lim _{t \rightarrow 0} t^{\frac{1}{2}} y(t)=\frac{1}{2} \int_{0}^{1} s^{-\frac{1}{2}} H(s, x(s), y(s)) d s \\
\lim _{t \rightarrow \frac{1}{2}^{+}}[t-1 / 2]^{\frac{1}{3}} x(t)=1 \\
\lim _{t \rightarrow \frac{1}{2}+}[t-1 / 2]^{\frac{1}{2}} y(t)=1 .
\end{array}\right.
$$

where

$$
\begin{aligned}
& f(t, x, y)=\left\{\begin{array}{l}
c_{1}+b_{1} t^{\frac{1}{3}} x+a_{1} t^{\frac{1}{6}} y^{\frac{1}{3}}, t \in(0,1 / 2], \\
c_{1}+b_{1}(t-1 / 2)^{\frac{1}{3}} x+a_{1}(t-1 / 2)^{\frac{1}{6}} y^{\frac{1}{3}}, t \in(1 / 2,1],
\end{array}\right. \\
& g(t, x, y)=\left\{\begin{array}{l}
c_{2}+b_{2} t x^{3}+a_{2} t^{\frac{1}{2}} y, t \in(0,1 / 2], \\
c_{2}+b_{2}(t-1 / 2) x^{3}+a_{2}(t-1 / 2)^{\frac{1}{2}} y, t \in(1 / 2,1],
\end{array}\right. \\
& G(t, x, y)=\left\{\begin{array}{l}
C_{1}+B_{1} t^{\frac{1}{3}} x+A_{1} t^{\frac{1}{6}} y^{\frac{1}{3}}, t \in(0,1 / 2], \\
C_{1}+B_{1}(t-1 / 2)^{\frac{1}{3}} x+A_{1}(t-1 / 2)^{\frac{1}{6}} y^{\frac{1}{3}}, t \in(1 / 2,1],
\end{array}\right. \\
& H(t, x, y)=\left\{\begin{array}{l}
C_{2}+B_{2} t x^{3}+A_{2} t^{\frac{1}{2}} y, t \in(0,1 / 2], \\
C_{2}+B_{2}(t-1 / 2) x^{3}+A_{2}(t-1 / 2)^{\frac{1}{2}} y, t \in(1 / 2,1],
\end{array}\right.
\end{aligned}
$$


with $c_{i}, b_{i}, a_{i}, C_{i}, B_{i}, A_{i}(i=1,2)$ being nonnegative numbers.

Proof. Corresponding to $\operatorname{BVP}(3), \alpha=\frac{2}{3}, \beta=\frac{1}{2}, \lambda=\mu=1, t_{1}=\frac{1}{2}, p(t)=q(t)=t^{-\frac{1}{4}}$, $\varphi(t) \psi(t)=\frac{1}{2} t^{-\frac{1}{2}}, \Phi(x)=x^{3}$ with $\Phi^{-1}(x)=x^{\frac{1}{3}}$, the supporting function of $\Phi$ is $\omega(x)=x^{3}$ and the supporting function of $\Phi^{-1}$ is $\nu(x)=x^{\frac{1}{3}}, I(t, x, y)=J(t, x, y)=1$.

It is easy to see that $\rho(t)=\frac{t^{-k} \nu\left(t^{\beta-1}\right)}{L}$ with $L=1$, and $k=0$, and

$$
\begin{aligned}
& f\left(t,\left(t-t_{k}\right)^{-\frac{1}{3}} x,\left(t-t_{k}\right)^{-\frac{1}{2}} y\right)=c_{1}+b_{1} x+a_{1} \Phi^{-1}(y), \\
& g\left(t,\left(t-t_{k}\right)^{-\frac{1}{3}} x,\left(t-t_{k}\right)^{-\frac{1}{2}} y\right)=c_{2}+b_{2} \Phi(x)+a_{1} y, \\
& G\left(t,\left(t-t_{k}\right)^{-\frac{1}{3}} x,\left(t-t_{k}\right)^{-\frac{1}{2}} y\right)=C_{1}+B_{1} x+A_{1} \Phi^{-1}(y), \\
& H\left(t,\left(t-t_{k}\right)^{-\frac{1}{3}} x,\left(t-t_{k}\right)^{-\frac{1}{2}} y\right)=C_{2}+B_{2} \Phi(x)+A_{2} y .
\end{aligned}
$$

It is easy to see that $\bar{C}_{1}=\bar{C}_{2}=1$ and $\bar{B}_{1}=\bar{B}_{2}=\bar{A}_{1}=\bar{A}_{2}=0$ with

$$
\begin{aligned}
& \left|I\left(1 / 2,(1 / 2)^{-\frac{1}{3}} x,(1 / 2)^{-\frac{1}{2}} y\right)\right| \leq \bar{C}_{1}+\bar{B}_{1}|x|+\bar{A}_{1} \Phi^{-1}(|y|), \\
& \left|J\left(1 / 2,(1 / 2)^{-\frac{1}{3}} x,(1 / 2)^{-\frac{1}{2}} y\right)\right| \leq \bar{C}_{2}+\bar{B}_{2} \Phi(|x|)+\bar{A}_{2}|y| .
\end{aligned}
$$

One sees that (C) and (D) hold. By computation, we get

$$
\begin{aligned}
\sigma_{1}=: & \max \left\{\Gamma(2 / 3) E_{2 / 3,2 / 3}(1) B_{1}+\left[\Gamma(2 / 3) E_{2 / 3,2 / 3}(1)^{2} \mathbf{B}(2 / 3,3 / 4)\right.\right. \\
& \left.\left.+E_{2 / 3,2 / 3}(1) \mathbf{B}(2 / 3,3 / 4)\right] b_{1}, E_{2 / 3,2 / 3}(1) \mathbf{B}(2 / 3,3 / 4) b_{1}\right\}, \\
\delta_{1}=: & \max \left\{\Gamma(2 / 3) E_{2 / 3,2 / 3}(1) A_{1}+\left[\Gamma(2 / 3) E_{2 / 3,2 / 3}(1)^{2} \mathbf{B}(2 / 3,3 / 4)\right.\right. \\
& \left.\left.+E_{2 / 3,2 / 3}(1) \mathbf{B}(2 / 3,3 / 4)\right] a_{1}, E_{2 / 3,2 / 3}(1) \mathbf{B}(2 / 3,3 / 4) a_{1}\right\}, \\
\sigma_{2}=: & \max \left\{\Gamma(1 / 2) E_{1 / 2,1 / 2}(1) B_{2}+\left[\Gamma(1 / 2) E_{1 / 2,1 / 2}(1)^{2} \mathbf{B}(1 / 2,3 / 4)\right.\right. \\
& \left.\left.+E_{1 / 2,1 / 2}(1) \mathbf{B}(1 / 2,3 / 4)\right] b_{2}, E_{1 / 2,1 / 2}(1) \mathbf{B}(1 / 2,3 / 4) b_{2}\right\}, \\
\delta_{2}=: & \max \left\{\Gamma(1 / 2) E_{1 / 2,1 / 2}(1) A_{2}+\left[\Gamma(1 / 2) E_{1 / 2,1 / 2}(1)^{2} \mathbf{B}(1 / 2,3 / 4)\right.\right. \\
& \left.\left.+E_{1 / 2,1 / 2}(1) \mathbf{B}(1 / 2,3 / 4)\right] a_{2}, E_{1 / 2,1 / 2}(1) \mathbf{B}(1 / 2,3 / 4) a_{2}\right\} .
\end{aligned}
$$

Then Theorem 3.1 implies that $\operatorname{BVP}(41)$ has at least one solution if

$$
\sigma_{1}<1, \quad 8 \frac{\delta_{1}^{3} \sigma_{2}}{\left(1-\delta_{1}\right)^{3}}+\delta_{2}<1 .
$$

Remark 4.1. It is easy to see that $\operatorname{BVP}(33)$ has at least one solution for sufficiently small $b_{i}, a_{i}, B_{i}, A_{i}(i=1,2)$ and any $c_{i}, C_{i}(i=1,2)$. 


\section{Conclusions}

In this paper, we discussed the existences of solution for two classes of initial value problems of nonlinear impulsive fractional differential models on half lines involving Riemann-Liouville fractional derivatives. The investigation shows that these results and methods are helpful for study in the nonlinear area and the numerical simulation, especially for study in the the numerical solution of a fractional differential equation with multiple base points with or without impulse effects.

The most important part of this study is to develop the idea of impulsive fractional models, which is a first of its kind.

Another important part is to demonstrate the application of the powerful mathematical tool (fixed point theorems in Banach spaces) for solving nonlinear fractional differential models.

Some problems considered in this paper can be improved under weaker conditions on the functions $f$ and $I, J$. Further studies are also located on seeking the numerical simulation of these models.

This paper contributes within the domain of impulsive fractional differential equations. The author strongly believes that the article will highly be appreciated by the researchers working in the field of fractional calculus and on fractional differential models.

\section{Acknowledgment}

Supported by the Natural Science Foundation of Guangdong province (No:S2011010001900) and the Foundation for High-level talents in Guangdong Higher Education Project.

\section{References}

[1] M. Belmekki, Juan J. Nieto, R. Rodriguez-Lopez, Existence of periodic solution for a nonlinear fractional differential equation, Boundary Value Problems, 2009(2009), Article ID 324561, doi:10.1155/2009/324561.

[2] J. J. Nieto, Maximum principles for fractional differential equations derived from Mittag-Leffler functions, Applied Mathematics Letters, 23(2010)1248-1251.

[3] J. J. Nieto, Comparison results for periodic boundary value problems of fractional differential equations, Fractional Differential Equations, 1(2011)99-104.

[4] Z. Wei, W. Dong, J. Che, Periodic boundary value problems for fractional differential equations involving a Riemann-Liouville fractional derivative, Nonlinear Analysis: Theory, Methods and Applications, 73(2010)3232-3238.

[5] Z. Wei, W. Dog, Periodic boundary value problems for Riemann-Liouville fractional differential equations, Electronic Journal of Qualitative Theory of Differential Equations, 87(2011)1-13.

[6] A. A. Kilbas, and J.J. Trujillo, Differential equations of fractional order: methods, results and problems-I, Applicable Analysis, 78(2001)153-192.

[7] A. Arara, M. Benchohra, N. Hamidi, and J. Nieto, Fractional order differential equations on an unbounded domain, Nonlinear Analysis, 72(2010)580-586. 
[8] A. A. Kilbas, H. M. Srivastava, J. J. Trujillo, Theory and applications of frational differential equations, Elsevier Science B. V. Amsterdam, 2006.

[9] S. Z. Rida, H.M. El-Sherbiny, and A. Arafa, On the solution of the fractional nonlinear Schrodinger equation, Physics Letters A, 372(2008)553-558.

[10] A. M. Nakhushev, The Sturm-Liouville problem for a second order ordinary differential equations with fractional derivatives in the lower terms, Dokl. Akad. Nauk SSSR, 234(1977)308-311.

[11] S. Zhang, The existence of a positive solution for a nonlinear fractional differential equation, J. Math. Anal. Appl. 252(2000)804-812.

[12] E. Kaufmann, E. Mboumi, Positive solutions of a boundary value problem for a nonlinear fractional differential equation, Electronic Journal of Qualitative Theory of Differential Equations, $3(2008) 1-11$.

[13] R. Dehghant and K. Ghanbari, Triple positive solutions for boundary value problem of a nonlinear fractional differential equation, Bulletin of the Iranian Mathematical Society, 33(2007)114.

[14] Y. Liu, Positive solutions for singular FDES, U.P.B. Sci. Series A, 73(2011)89-100.

[15] S. Zhang, Positive solutions for boundary-value problems of nonlinear fractional differential equation, Electron. J. Diff. Eqns. 36(2006)1-12.

[16] Y. Zhao, S. Sun, Z. Han, M. Zhang, Positive solutions for boundary value problems of nonlinear fractional differential equations, Applied Mathematics and Computation, 217(2011)6950-6958.

[17] M. Benchohra, J. Graef, S. Hamani, Existence results for boundary value problems with nonlinear frational differential equations, Applicable Analysis, 87(2008)851-863.

[18] X. Wang, C. Bai, Periodic boundary value problems for nonlinear impulsive fractional differential equations, Electronic Journal of Qualitative Theory and Differential Equations, 3(2011)113.

[19] J. Mawhin, Topological degree methods in nonlinear boundary value problems, in: NSFCBMS Regional Conference Series in Math., American Math. Soc. Providence, RI, 1979.

[20] G. L. Karakostas, Positive solutions for the $\Phi$-Laplacian when $\Phi$ is a sup-multiplicative-like function, Electron. J. Diff. Eqns., Vol. 68(2004)1-12.

[21] K. S. Miller, S. G. Samko, Completely monotonic functions, Integr. Transf. Spec. Funct., 12(2001)389-402.

[22] M. Belmekki, J. Nieto, R. Rodrguez-Lopez, Existence of solution to a periodic boundary value problem for a nonlinear impulsive fractional differential equation, Electron. J. Qual. Theory Differ. Equ. 2014, No. 16, 1-27. 